\title{
Ceñlstán
}

\section{Representação de patrimônios culturais em plataformas digitais: o modelo de dados da Europeana}

\author{
Maria Lígia Triques \\ Mestranda; Universidade Federal de São Carlos, SP, Brasil; \\ ligia.triques@gmail.com \\ Ana Carolina Simionato Arakaki \\ Doutora; Universidade Federal de São Carlos, SP, Brasil; \\ acsimionato@ufscar.br
}

\begin{abstract}
Resumo: Com a finalidade de representar de forma abstrata as relações e entidades, os modelos de dados ganham destaque como melhores práticas nos processos de análise e representação da informação, especificamente no planejamento e desenvolvimento de sistemas interoperáveis e persistentes. Nesse sentido, apresenta-se um estudo sobre o Europeana Data Model (EDM), modelo de dados desenvolvido pela plataforma Europeana com base nas tecnologias semânticas e nos princípios do Linked Open Data. Por meio de uma pesquisa qualitativa, exploratória, bibliográfica e documental, discute-se como o uso de um modelo de dados, tal como o EDM, possibilita que as necessidades de representação informacional de coleções de dados de patrimônios culturais sejam atendidas no ambiente $W e b$. Desse modo, o objetivo do estudo foi analisar a estruturação e representação proposta pela Europeana para as coleções de patrimônios culturais. Como resultado, destaca-se a modelagem de dados, que é o processo pelo qual o EDM pauta seu funcionamento, possibilitando que as necessidades informacionais sejam contempladas. Conclui-se, por fim que a importância dos modelos de dados, tal como o EDM, encontra-se na possibilidade de apreender o contexto semântico a qual um conjunto de dados de patrimônios culturais pertence ou está relacionado, garantindo a persistência de seus conceitos e relações nos ambientes informacionais.
\end{abstract}

Palavras-chave: Modelo de Dados. Metadados. Patrimônio Cultural. Europeana. EDM.

\section{Introdução}

Os dados podem ser definidos como expressão mínima de um conteúdo e podem ser produzidos de inúmeras formas, seja por humanos ou por máquinas, eles estão presentes em todas possibilidades de produção de recursos informacionais. Os dados também assumem a função de especificar características e descrever outros dados, definindo a forma com eles serão utilizados, exibidos e 
contextualizados (SOUZA; ALVARENGA, 2004), sendo nesse caso, denominados como metadados.

Os profissionais da informação têm longa história de criação e compartilhamento de metadados (RILEY, 2017), trabalhando com esses elementos descritivos e contextuais também de forma independe do ambiente digital. Como explica Alves (2010), os metadados configuram-se como uma denominação atual no processo de tratamento da informação, se referindo a elementos que permitem a construção de representações para um recurso informacional.

Os dados vêm se multiplicando à medida que o desenvolvimento das tecnologias permite maiores possibilidades de compartilhamento e utilização de diversos tipos de conteúdo nos ambientes digitais. Nesse cenário, diversos domínios do conhecimento se deparam com a intensa produção de dados, modificando as dimensões da atuação humana, em especial nos setores relacionados com a produção e disseminação de informação e conhecimento. É o caso do domínio dos patrimônios culturais que tem direcionado seus esforços para estruturar suas coleções e compartilhá-las, principalmente por meio do ambiente Web (HYVÖNEN, 2012).

Composto principalmente pelas instituições como bibliotecas, arquivos, e museus, o domínio de patrimônios culturais agrega um rico corpo de conhecimento que resulta em importante recursos informacionais. Os recursos de patrimônios culturais referem-se:

[...] ao legado de objetos físicos, meio ambiente, tradições e conhecimento de uma sociedade, herdado do passado, mantido e desenvolvido no presente e preservado (conservado) para o benefício das gerações futuras. (HYVÖNEN, 2012, p.1, tradução nossa).

Grande parte da memória social e da história da humanidade podem ser apreendidas por meio desses legados que constituem importantes fontes de informação e conhecimento. Tais legados tem sido cada vez mais encontrados em formatos digitais, a partir da publicação de seus dados, sendo produzidos, disponibilizados e compartilhados de forma aberta e distribuída no ambiente Web. 
Um grande exemplo é a Europeana (disponível em: http://www.europeana.eu/), também chamada de Biblioteca Digital Europeana. Uma plataforma digital que reúne e estrutura dados de diversas instituições de patrimônios culturais, não só disponibilizando esse conteúdo de modo estruturado por meio da interface Web, como também, promovendo o reuso e o acesso aberto dos dados por meio de seu modelo de dados, o Europeana Data Model (EDM).

Modelos de dados ampliam as possibilidades de organizar, compartilhar e processar as informações sobre patrimônios culturais em meio digital, o que os coloca em posição de destaque no âmbito da Ciência da Informação, em especial, no que se refere ao tratamento de dados que estão alocados em ambientes informacionais direcionados pela construção semântica e pela ligação de dados. Contudo, garantir a persistência dos aspectos semânticos dos recursos de patrimônios culturais não é uma tarefa simples, principalmente considerando os desafios próprios da organização e representação de patrimônios culturais.

Diante desse cenário, questiona-se: como um modelo de dados, tal como o EDM, pode viabilizar que as necessidades de representação de coleções heterogêneas de patrimônios culturais sejam atendidas no ambiente Web?

A partir da contextualização teórica e considerando a documentação do EDM, o objetivo do trabalho consiste em analisar a estruturação e representação proposta pela Europeana para a contextualização semântica das coleções de patrimônios culturais.

A pesquisa é classificada como qualitativa e exploratória. Aos procedimentos técnicos recorreu-se a pesquisa bibliográfica, com 0 levantamento realizado em nível nacional e internacional, em fontes de pesquisa primárias, secundárias e terciárias. Para um maior embasamento, utilizou-se a documentação elaborada e disponibilizada pela própria plataforma Europeana.

Em relação ao levantamento bibliográfico, o período selecionado para a pesquisa contempla publicações a partir de 2005, ano em que se observa o início das publicações sobre a temática. As fontes utilizadas no levantamento bibliográfico foram: Base de Dados Referenciais de Artigos de Periódicos em Ciência da Informação (BRAPCI), Portal de Periódicos da Capes, Google 
Acadêmico, Scientific Electronic Library Online (SciELO), Scopus e Web of Science, tendo como resultados artigos, trabalhos em eventos, livros e capítulo de livros. A análise da literatura cientifica e da documentação fornecida pela plataforma, permitiu observar as potencialidades e os desafios da plataforma Europeana e de seu modelo de dados, o Europeana Data Model (EDM) no que tange o objetivo proposto.

\section{Os modelos de dados e a representação na Web}

Nos últimos anos o aumento da oferta de informações, principalmente em meio digital, levou ao crescimento da busca por instrumentos e métodos mais eficazes para a organização e a representação da informação e do conhecimento. Em conjunto com o contínuo desenvolvimento das Tecnologias da Informação e Comunicação (TIC), diferentes abordagens têm se apresentado ao âmbito da Ciência da Informação para buscar atender as necessidades do tratamento de crescentes volumes de dados.

Nesse cenário, a Web é considerada como uma das principais fontes para disponibilizar e obter informações em meio digital. Nas últimas décadas, a chamada Web Semântica, extensão da Web (BERNERS-LEE; HENDLER; LASSILA, 2001), tem se estabelecido a partir de conjunto de várias tecnologias, possuindo como base os metadados para a representação dos recursos informacionais. Tais tecnologias, conhecidas como tecnologias semânticas, fornecem a padronização nas estruturas de dados, que vão envolver linguagens para estruturação, padrões de representação e interoperabilidade (ALVES, 2005; SOUZA; ALVARENGA, 2004).

A padronização destaca-se pela garantia da construção de uma linguagem comum e compartilhada entre os componentes da Web, permitindo explicitar o sentido agregado aos dados, e assim, estabelecer as ligações de significado entre eles de tal forma que as máquinas tenham maior capacidade de processar e trocar informações semanticamente.

Dentre as principais tecnologias semânticas que auxiliam no funcionamento da Web, encontram-se a Extensible Markup Language (XML), o Resource Description Framework (RDF), o Simple Knowledge Organization 
System (SKOS), o Uniform Resource Identifier (URI), o Ontology Web Language (OWL) e suas derivações. Atuando em conjunto a essas tecnologias, estão os princípios Linked Data que consistem em “[...] um conjunto de práticas para publicar e conectar dados estruturados na Web [...]" (CUNHA; SOUZA; LÓSCIO, 2011, p. 79) com o intuito de que os conjuntos de dados sejam coerentemente descritos, persistidos e conectados. Alinhado a proposta do Linked Data, está o Linked Open Data, que se refere aos dados que são vinculados sob licença aberta. A fusão desses princípios origina o Linked Open Data (LOD) que permite melhorar a conectividade e a recuperação dos dados.

Desse modo, ressalta-se que a descrição e estruturação de dados é um fator imprescindível para o funcionamento de qualquer ambiente informacional, principalmente no contexto da $W e b$, no qual diferentes esquemas ou padrões são necessários e usados para os diferentes tipos de recursos digitais nas plataformas que lidam com conteúdo de diferentes domínios (HYVÖNEN, 2012).

Para tanto, a Web se tornou objeto de estudo bastante discutido no âmbito da Ciência da Informação, envolvendo questões de estruturação sintática e semântica, bem como de ligação entre dados no processo de representação da informação. Ao longo dos anos, estudos têm abordado essas questões a partir de diversos instrumentos como padrões e esquemas de metadados, vocabulários controlados, tesauros e ontologias. No entanto, a existência de uma variedade desses instrumentos gera diferentes formatos para os dados, resultando em estruturas e terminologias conflitantes (CASTRO; SIMIONATO; ZAFALON, 2016). Esse fato dificulta a interoperabilidade, que é a capacidade de vários sistemas trocarem dados com a mínima perda de conteúdo e funcionalidade (RILEY, 2017).

Em relação a interoperabilidade dos dados de patrimônios culturais observa-se que a grande dificuldade está relacionada com a heterogeneidade semântica, caracterizada pelos conflitos na interpretação da informação (CASTRO; SIMIONATO; ZAFALON, 2016). Isso ocorre principalmente pela natureza desses dados que são derivados de itens “[...] sintática e semanticamente heterogêneos, multilíngues, semanticamente ricos e altamente interligados.” (HYVÖNEN, 2012, p. 8, tradução nossa). As soluções para a 
questão da interoperabilidade apontam para os sistemas de harmonização de dados, baseados em modelos conceituais e ontologias (DOERR, 2003; HYVÖNEN, 2012).

Modelos conceituais de dados são abstrações do mundo real desenvolvidos e planejados para representarem um domínio. Coyle (2016) salienta que se pode atribuir às bibliotecas o desenvolvimento de alguns dos primeiros modelos de dados com a criação dos catálogos de cartões. Com a evolução da tecnologia, outros modelos surgiram a partir da possibilidade de armazenar dados em sistemas computacionais.

Fusco (2010) destaca a importância dos estudos relacionados aos modelos de dados para o cenário da representação da informação, uma vez que eles viabilizam uma descrição mais ampla e efetiva da informação por meio da modelagem conceitual de dados. Esta, por sua vez, pode ser definida como “[...] um processo de abstração que tem como objetivo identificar, modelar e implementar um modelo de dados consistente com as necessidades do domínio [...]" (FUSCO, 2010, p. 95).

$\mathrm{Na} W e b$, os modelos de dados estão baseados nos padrões recomendados internacionalmente pela World Wide Web Consortium (W3C), consórcio mundial responsável pela Web, sendo o Resource Description Framework (RDF) a recomendação para a publicação e vinculação dos dados (RDF WORKING GROUP, 2014).

Os modelos de dados caracterizam-se pela possibilidade de identificar as propriedades essenciais de um domínio (LISBOA FILHO; IOCHPE, 1999), ou seja, eles permitem apreender os aspectos semânticos desse domínio. Portanto, os modelos de dados são uma forma de representação utilizada para descrever um conjunto de dados e seus relacionamentos, bem como as regras para manipular esses dados em um dado domínio (FUSCO, 2010). Sendo assim, os modelos de dados ainda podem ser entendidos como ferramentas que fornecem estrutura a um determinado conteúdo, permitindo a interação entre suas partes e o estabelecimento de diversas conexões semânticas.

Nesse contexto, um importante instrumento para descrever modelos conceituais no âmbito da representação de recursos informacionais na Web são 
as ontologias (RAMALHO, 2006). De acordo com Santarem Segundo (2015, p. 226) as ontologias são uma forma

[...] de se construir uma relação organizada entre termos dentro de um domínio, favorecendo a possibilidade de contextualizar os dados, tornando mais eficiente e facilitando o processo de interpretação dos dados [...].

Em outras palavras, as ontologias podem ser entendidas como uma forma de explicitação computacional da representação de conceitos e de seus relacionamentos dentro de um domínio.

As ontologias permitem a representação de recursos informacionais na Web por meio de declarações que explicitam o significado e as relações desses recursos de modo a criar uma interpretação integrada dos dados. Isso é possível pois as ontologias podem ser utilizadas na integração de esquemas de metadados, formando um esquema global para o mapeamento de diferentes fontes de metadados (CASTRO; SIMIONATO; ZAFALON, 2016). Cruz e Xiao (2005, p. 3, tradução nossa), compreendem que "[...] a integração de dados semânticos é o processo de usar uma representação conceitual dos dados e de suas relações para eliminar possíveis heterogeneidades." Por essa razão, as ontologias são consideradas uma das principais estruturas da Web Semântica, configurando-se como instrumentos que permitem a descrição e integração dos recursos informacionais.

Diante disso, verifica-se que no intuito de disponibilizar suas coleções e contribuir com as redes de informações digitais, as instituições que trabalham com o patrimônio cultural, tem buscado as tecnologias semânticas para trabalhar com seus conjuntos de dados no ambiente Web.

Liu, Bikakis e Vlachidis (2017) destacam que o volume, a diversidade e a heterogeneidade dos recursos informacionais de patrimônios culturais que estão em museus, galerias de arte e outras instituições, contribuiu para que muitas dessas instituições procurassem por modelos de dados para organizar e representar melhor suas coleções e desenvolver serviços aperfeiçoados para os visitantes em meio digital. 
A Europeana é um exemplo disso, apresentando uma plataforma que dá acesso a um vasto conteúdo sobre o patrimônio cultural, oferecendo ainda um detalhado programa para disponibilização de dados com recursos de agregação e contextualização semântica (DOERR et al, 2010).

A iniciativa Europeana teve início em 2005, motivada pelo projeto de reunir em um único lugar o vasto conteúdo sobre patrimônios culturais da Europa. Sua proposta norteadora consistia em criar "[...] um espaço no qual todas as manifestações da herança cultural e científica da Europa pudessem ser conectadas e integradas em um único portal, em um ambiente multilíngue." (PURDAY, 2009, p. 920, tradução nossa).

Após alguns anos de testes e aprimoramentos, com foco no processo de aquisição de conteúdo e preparação de dados, a Europeana tornou-se em 2010 um sistema operacional. No mesmo ano houve a criação de um comitê denominado de "Comitê dos Sábios", que tinha o objetivo de desenvolver recomendações referentes a disponibilização, digitalização, acesso e preservação do conteúdo (WINER; ROCHA, 2013). Com isso, a Europeana ganhou força e novos apoiadores, chegando a ter, em novembro de 2010, um acervo de mais de 25 milhões de registros (WINER; ROCHA, 2013). Atualmente, a plataforma conta com mais de 58 milhões de itens, como livros, sons, vídeos, obras de arte e coleções temáticas especiais que incluem arte, moda, música, fotografia e a documentos da Primeira Guerra Mundial.

Nota-se, portanto, a amplitude que tal iniciativa, hoje em pleno funcionamento e expansão, vem tomando, colocando-se como principal fonte de informação sobre patrimônio cultural na Europa. Tal como a Europeana, demais iniciativas surgem com o apoio do governo, como é o caso da Digital Public Library of America (DPLA) que busca reunir e estruturar o conteúdo sobre o patrimônio cultural dos Estados Unidos (EUA), com uma proposta bastante semelhante à da Europeana. Outro exemplo é a DigitalNZ, criada a partir da proposta de conectar o conteúdo digital da Nova Zelândia que foi implementada pela a Biblioteca Nacional e pelo governo do país, com um programa próprio de agregação e contextualização de dados chamado Supplejack. 
Operando cada uma a seu modo, as três plataformas tem em comum a proposta de integrar, estruturar e disseminar dados de patrimônios culturais na Web por meio de modelos de dados apoiados nas tecnologias semânticas.

Liu, Bikakis e Vlachidis (2017, p. 344, tradução nossa) explicam que para o domínio dos patrimônios culturais, “[...] as tecnologias da Web Semântica são usadas principalmente para duas finalidades: o desenvolvimento de sistemas de curadoria interna e o estabelecimento de bancos de dados abertos de coleta." Em ambos os casos, as tecnologias semânticas permitem a padronização dos metadados que descrevem as entidades relativas aos patrimônios culturais e seus relacionamentos, possibilitando, dessa forma, a interoperabilidade e a sustentabilidade para diferentes coleções de dados.

Por essa razão, ressalta-se a importância de se levar em consideração a capacidade de interoperar e de persistir ao longo do tempo nos processos de representação da informação, em especial nos ambientes informacionais que lidam com grandes volumes de dados heterogêneos na Web.

\section{A representação da informação na plataforma na Europeana}

Sendo pioneira no projeto de reunir e estruturar dados de diversas instituições do patrimônio cultural, a Europeana pode ser considerada o principal exemplo de como as tecnologias semânticas auxiliam no processo de tratamento da informação de patrimônios culturais em meio digital. Além disso, seu detalhado programa de agregação e contextualização semântica permite verificar como as necessidades de representação de coleções heterogêneas de patrimônios culturais podem ser atendidas no ambiente Web.

Essas necessidades de representação estão vinculadas à natureza especifica dos recursos de patrimônios culturais, que apresentam dados heterogêneos, semanticamente ricos, multilíngues e altamente interligados. Essa riqueza e variedade de conteúdo traz naturalmente desafios para o tratamento informacional, implicando diretamente na representação da informação. Essa representação, por sua vez, deve contemplar não só a descrição com vistas a recuperação, mas também a sustentabilidade e a interoperabilidade para poder apoiar processos distribuídos de criação e compartilhamento de conteúdo. 
Como explicam Concordia, Gradmann e Siebinga (2009) o projeto europeu foi um esforço para construir uma plataforma aberta que promovesse a interoperabilidade funcional, técnica e de dados. Nesse sentido, a interoperabilidade entre domínios foi um princípio fundamental a ser levado em consideração desde o início do projeto, partindo da "[...] ideia de que a colaboração e a transferência de conhecimento entre museus, bibliotecas, arquivos e coleções audiovisuais era fundamental para o sucesso do empreendimento" (PURDAY, 2009, p. 921, tradução nossa). Por essa razão, no decorrer do desenvolvimento do projeto e ainda hoje, as questões relacionadas a interoperabilidade dos dados são cuidadosamente estudadas, levando em consideração padrões, princípios, vocabulários e requisitos de usuários.

Além disso, seu grande potencial está na possibilidade de disseminar a terceiros seus conteúdos por meio das Application Programming Interface $(\mathrm{API})^{1}$ específicas, permitindo amplo reuso de seus dados estruturados (MARCONDES, 2016). Essa tecnologia propicia que a Europeana ofereça um serviço de acesso remoto às suas coleções e que diversas comunidades façam uso de suas estruturas de representação de dados.

A construção da Europeana se alicerça na adoção de tecnologias e no desenvolvimento de padrões. Para a estruturação e representação de seus dados, a Europeana desenvolveu uma ferramenta que permite mapear e enriquecer metadados denominada como Europeana Data Model (EDM). (EUROPEANA, 2013).

O EDM configura-se como um modelo de dados fundamentado nas tecnologias da Web Semântica e nos princípios do Linked Open Data. Foi desenvolvido a partir de um esforço colaborativo entre os setores do patrimônio cultural para suportar extensões e refinamentos, a fim de acomodar as particulariedades dos dados dos vários setores que compõe o domínio dos patrimônios culturais (CHARLES; ISAAC, 2015).

Cada um dos diferentes setores de patrimônio cultural - museus, arquivos, galerias, bibliotecas - que fornecem dados para a Europeana tem seus respectivos padrões para os dados o que lhes cofere diferentes formatos. 
Como destaca Petras et al (2017), historicamente, arquivos, bibliotecas e museus seguem diferentes práticas de representação do conteúdo, criadas a partir de estruturas especificas que são difíceis de transformar em um formato interoperável. Como resultado, as coleções agregadas da Europeana diferem na quantidade, qualidade e especificidade das representações. Por isso, o EDM foi desenvolvido para executar uma cobertura total dos dados, integrando os metadados dos vários setores. Assim sendo, o EDM não se baseia em nenhum padrão comunitário específico, mas adota uma estrutura aberta baseada na Web Semântica que pode acomodar a variedade e a riqueza de padrões específicos de cada setor (EUROPEANA, 2013).

Isso torna o modelo capaz de reunir e estruturar os dados de modo que várias representações coexistam para um mesmo item fornecido, o que é possivel devido ao fato do EDM permitir o enriquecimento de dados por meio de uma variedade de fontes externas.

Como explicam Coneglian e Santarem Segundo (2017, p. 91), o EDM “[...] utiliza diversos vocabulários para formar um modelo que consiga expressar as ligações existentes, entre autores, obras, organizações, direitos autorais, além de outros tipos de informações, contidas em um objeto cultural." Por essa razão, o EDM permite a integração dos dados fornecidos a Europeana, que são estruturados a partir de diferentes perspectivas, permitindo ampliar e aprimorar as formas de interação entre a plataforma e cada comunidade usuária.

Doerr et al (2010, p. 6-7, tradução nossa) explicam que:

O EDM não vincula a representação de metadados ingeridos a um esquema comum, mas age como uma ontologia de nível superior comum, de acordo com a qual os metadados compatíveis com outros modelos de dados originais podem ser expressos.

Sendo assim, o EDM pode ser considerdo como uma âncora, que permite conectar vários modelos mais refinados, ao mesmo tempo em que garante a interoperabilidade em um nível semântico (CHARLES; ISAAC, 2015). Um exemplo de modelo que está alinhados ao EDM, é o CIDOC-CRM (CIDOC Conceptual Reference Model), principal ferramenta para a modelagem de dados no campo do patrimônio cultural. Esse alinhamento funcional com demais 
modelos e vocabulários, faz com que o EDM suporte uma descrição mais completa do conteúdo ao mesmo tempo em que se preocupa com a definição de perfis de aplicação ${ }^{2}$ para que os dados sejam adequados a cada finalidade.

A partir da abordagem por perfis de aplicação, o EDM faz mais do que mesclar e modificar os elementos existentes, ele cria estruturas modulares ${ }^{3}$ que permitem integrar e expandir vários vocabulários já existentes, acrescentando ainda seus próprios metadados. Seguindo o princípio da modularidade, o modelo reutiliza elementos de metadados externos e se alinha a outros modelos conceituais, possibilitando criar perfis de aplicação especificos e enriquecidos por diferentes provedores de dados (ZENG; QIN, 2016).

Sendo assim, o EDM é capaz de mapear várias fontes e integrar diversos tipos de dados considerando as difrentes perspectivas e necessidades de informação, multidimensionando as formas de interação entre plataforma e cada comunidade usuária.

O modelo tem como objetivo, portanto, ser um meio de integração para coletar, conectar e enriquecer as descrições fornecidas pelos provedores de conteúdo da Europeana, permitindo incluir qualquer elemento descritivo pertinente a representação. Nesse processo, destacam-se os princípios do Linked Open Data, uma vez que a publicação de dados conectados de forma aberta permite a integração e a retutilização de dados, otimizando a representação da informação e ampliando as possibilidades de colaboração.

Como discute Marcondes (2018), tanto o processo de publicação, quanto o de representação da informação, usando Linked Open Data (LOD), permite criar o que o autor denomina como 'links semânticos', que são os possíveis relacionamentos culturalmente relevantes que as coleções do patrimônio cultural estabelecem entre si, a partir da relação entre seus dados.

Com a representação desses relacionamentos culturalmente significativos, é possível apresentar o contexto semântico a que cada recuso informacional do patrimônio cultural pertence ou está relacionado, abrangendo desde as descrições, relacionamentos e interpretações sobre os recursos de patrimonios cultutais, até os atores, locais e eventos envolvidos em sua produção e operação. 
O processo de representação em EDM inicia-se no momento da obtenção dos dados, no qual eles são convertidos em recursos representados pelo padrão RDF/XML, recebendo um Uniform Resource Identifier (URI) para um identificação percistente na Web. Em seguida, é realizado o processo de enriquecimento dos dados, em que são inseridos, pela própria Europeana, demais dados relevantes e pertinentes sobre os recursos. Esses dados podem ser vinculados a partir de URIs já existentes em outras bases, o que lhes confere maior representatividade (CONEGLIAN; SANTAREM SEGUNDO, 2017).

Entende-se, portanto que o EDM busca enriquecer a representação dos dados a partir de instrumentos como vocabulários controlados e ontologias de domínio, de modo a garantir um maior nível de interação, integração e precisão na recuperação da informação ao mesmo tempo em que propicia a continua atualização dos dados em conformidade com as comunidades interessadas.

Para alcançar tal objetivo, o EDM está baseado em diretrizes e padrões reconhecidos internacionalmente, como Open Archives Initiative Object Resue \& Exchange (OAI-ORE), o Dublin Core (DC), Simple Knowledge Organization System (SKOS) e Uniform Resource Identifier (URI) (HYVÖNEN, 2012; DOERR et al, 2010; ARAKAKI, 2016), que vão auxiliar na tarefa de representar os dados individualmente. Além disso, uma das principais ferramentas que possibilita o funcionamento do EDM é o Resource Description Framework (RDF), que

[...] permite representar informações estruturadas sobre qualquer recurso na forma de simples instruções triplas (sujeito, predicado, objeto). A visão que guia o RDF é que os recursos podem ser descritos por meio de conexões semanticamente significativas entre eles (DOERR et al., 2010, p.3, tradução nossa).

Por meio dessa abordagem em triplas, é possível criar ligações semânticas que transmitem um conhecimento significativo processável por maquinas. Por essa razão, Hyvönen (2012, p. 45, tradução nossa) entende que o EDM configura-se como "[...] uma estrutura conceitual ou ontologica em que modelos mais específicos podem ser anexados e aprimorados para garantir a interoperabilidade entre eles". 
A partir das declarações em RDF, é possível definir classes e propriedades em formato de triplas (sujeito, predicado, objeto). Essa definição apresenta-se em uma hierarquia de classes, como ilustrado na figura 1, que demonstra como são definidos os relacionamentos entre os dados no EDM.

Figura 1 - Hierarquia de classes do EDM

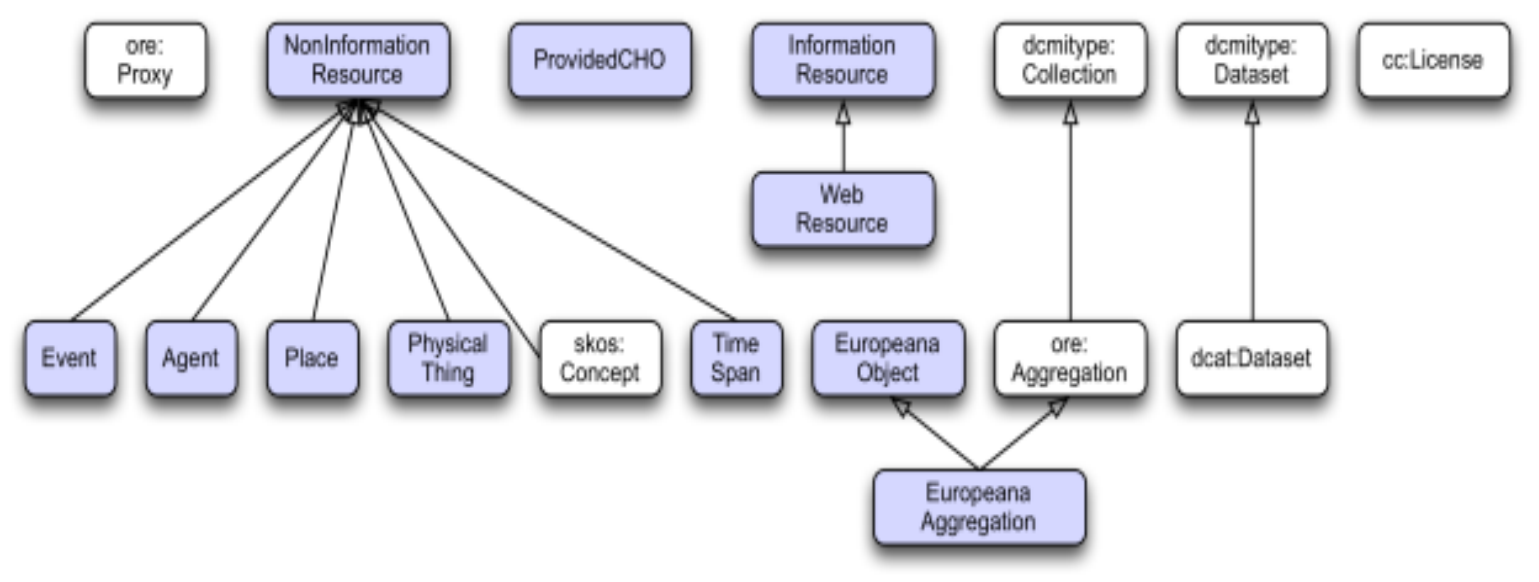

Fonte: Europeana (2017).

A figura 1 demonstra as relações que o EDM estabelece ao ligar-se a elementos já existentes, reutilizando vocabulários internacionalmente reconhecidos ao em vez de criar novos. As classes do EDM são representadas pelos retângulos azuis e as classe reutilizadas de outros padrões, pelos retângulos brancos. Coneglian e Santarem Segundo (2017, p. 91) explicam que

O uso e integração de vocabulários internacionalmente reconhecidos e nascidos de padrões de metadados já sedimentados, torna o EDM um rico modelo de descrição dos objetos da Europeana, fortalecendo a estrutura de descrição semântica dos recursos.

Para o processo de estruturar os dados, o EDM possui três classes principais de recursos que são provenientes do pacote de dados que deve ser fornecido à Europeana. São elas:

- o "recurso de patrimônio cultural fornecido" em si (uma pintura, um filme, uma partitura musical, um livro...) (edm: ProvidedCHO) 
- uma ou mais representações digitais acessíveis deste recurso, algumas das quais serão usadas como visualizações (a imagem digital da pintura.) (edm: WebResource)

- uma agregação para representar o resultado da atividade desse provedor. (ore: Aggregation) (EUROPEANA, 2013, p.10, tradução nossa).

O elemento ProvidedCHO, se refere ao recurso original que está sendo descrito, o elemento WebResource diz respeito às representações digitais dos recursos que estão na Web e o elemento Aggregation, vincula os elementos, fornecendo dados sobre a origem da descrição. Ainda, existem classes que podem ser agregadas para representar os recursos contextuais de cada elementos específico, tais como: Agent, Place, TimeSpan, Concept, Event e PhysicalThing. (EUROPEANA, 2013).

A atribuição desses elementos descritivos permite fornecer uma representação rica, que não exclui outras já existentes, ao contrário, agrega mais descrições para melhorar a representação do recurso de patrimônio cultural em seu domínio, enriquecendo a contextualização semântica. Portanto, vincular metadados ajuda a contextualizar semanticamente os recursos de patrimônio cultural e facilita a sua recuperação.

$\mathrm{Na}$ figura 2, pode-se vizualizar como ocorre esse enriquecimento dos dados no EDM, fornecendo um panorama da relação entre as principais classes e propriedades que as ligam. 
Figura 2 - Representação em EDM da pintura Mona Lisa, de Leonardo da Vinci

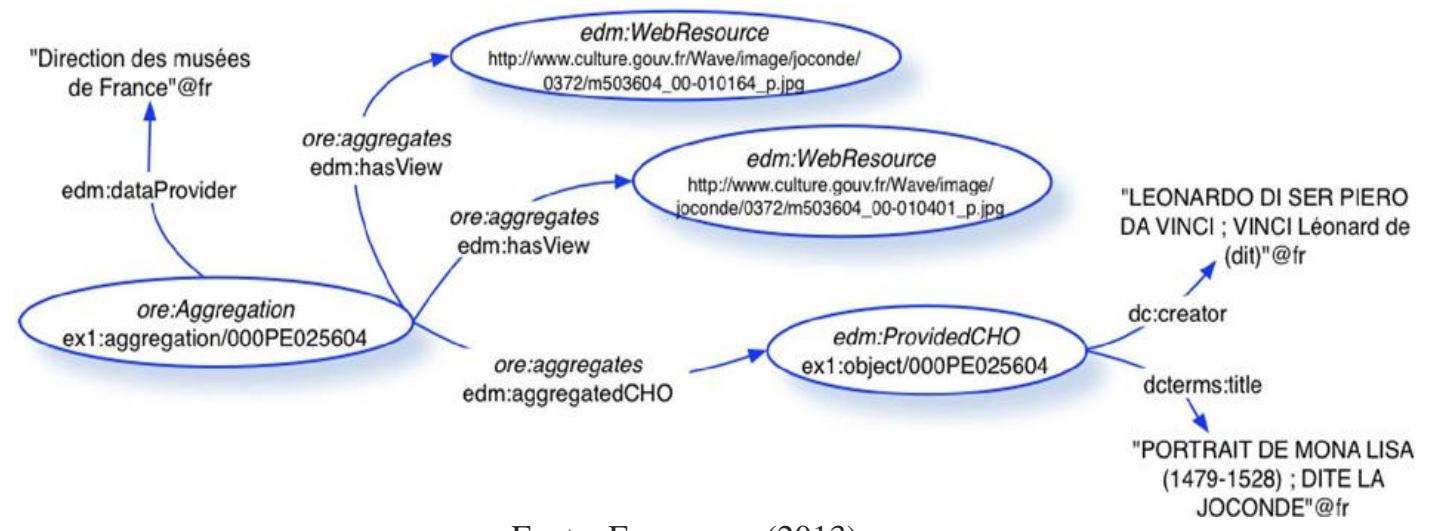

Fonte: Europeana (2013).

No exemplo, a obra Mona Lisa, de Leonardo da Vinci é representada pelo 'edm: ProvidedCHO' e está relacionada a outras propriedades, como 'ore: Aggregation', que indica os dados do seu provedor (edm:dataProvider), a 'Direction des Musées de france'. Ainda, a obra é relacionada, por meio da atribuição de elementos descritivos como 'dc: criator' e 'dc: title', ao seu criador e ao seu título, ambos com valores atribuídos pela vinculação de dados aberto de outras bases de dados. Além disso, por meio das subpropriedades 'ore: agregates', a obra pode ser vinculada a outras representações digitais que já estão na Web, indentificadas pelo elemento 'edm:WebResource' (EUROPEANA, 2013).

Diante disso, retoma-se a importância da modelagem conceitual de dados. Como visto, a modelagem de dados é o processo pelo qual ocorre a abstração dos elementos de um domínio, em que são considerados seus aspectos semânticos essenciais. Por meio desse processo é que o EDM pauta seu funcionamento, possibilitando que as necessidades informacionais dos recursos sejam contempladas. A modelagem do EDM está baseada nos seguintes requisitos:

- R1: distinção entre "objetos fornecidos" (pintura, livro, filme, sítio de arqueologia, documentos de arquivos, etc.) e suas representações digitais;

- R2: distinção entre objetos e registros de metadados que descrevem um objeto; 
- R3: vários registros para o mesmo objeto devem ser permitidos, contendo declarações potencialmente contraditórias sobre esse objeto;

- R4: suporte para objetos que são compostos de outros objetos;

- R5: compatibilidade com diferentes níveis de abstração de descrição (por exemplo, se um fornecedor deseja enviar descrições que seguem as distinções introduzidas no FRBR Group 1 [FRBR]);

- R6: o EDM fornece um formato padrão de metadados que pode ser especializado;

- R7: suporte para recursos contextuais, incluindo conceitos de vocabulários controlados (EUROPEANA, 2013, p. 8, tradução nossa).

Pautando-se nesses requisitos, o EDM explorara a riqueza e a variedade dos dados disponíveis na Web sobre patrimônio cultural para uma representação mais robusta, apoiando a integração dos vários modelos e padrões de modo que todas as descrições originais possam ser coletadas e conectadas por meio de conceitos de nível superior (EUROPEANA, 2013). Isso é notável nos três 'princípios de design' nos quais o modelo está fundamentado:

- D1: O EDM permite a integração de dados em um ambiente aberto: é impossível antecipar todos os dados contribuídos;

- D2: O EDM permite uma funcionalidade rica, possivelmente por meio de extensões;

- D3: O EDM deve reutilizar os modelos existentes (padrão) tanto quanto possível (EUROPEANA, 2013, p. 8, tradução nossa).

Para que esses princípios sejam seguidos, os dados que incorporam a Europeana precisam atender a requisitos mínimos de qualidade. Por isso, todos os dados fornecidos pelos provedores devem seguir critérios de publicação definidos pela plataforma, visando garantir a qualidade e aumentar a possibilidade de conexão entre as coleções. Para tanto, o grupo Europeana desenvolveu o Europeana Publishing Guide, (disponível em: https://pro.europeana.eu/post/publication-policy), um conjunto de diretrizes que descreve os requisitos mínimos de metadados necessários para incorporar dados à Europeana. Desse modo, a plataforma auxilia os agregadores e parceiros de dados a compartilharem seus dados em formatos padronizados e interoperáveis, buscando garantir que os dados sejam autênticos e confiáveis (EUROPEANA, 2019). Os mesmos critérios são aplicáveis aos materiais anteriormente publicados na Europeana, mantendo apenas os metadados que estão em 
conformidade com os requisitos definidos, buscando assim, garantir uma melhoria consistente na qualidade.

Porém, essa não é uma tarefa trivial, pois, como visto, diferentes instituições do patrimônio cultural seguem diferentes abordagens para representar e gerenciar seus acervos e coleções, o que dificulta a uniformidade, tanto dos formatos, quanto da qualidade desses dados.

Portanto, percebe-se que um dos grandes desafios da plataforma deriva do processo de agregação de dados que consiste em um conjunto de ações que envolvem desde a criação e entrega dos dados, até a definição do mapeamento, normalização e enriquecimento, no qual os dados são definidos e redefinidos de acordo com diferentes padrões e preparados para atender diferentes ambientes informacionais e consumidores (EUROPEANA, 2019).

A qualidade variável dos dados que são fornecidos acaba por dificultar essa longa cadeia de ações afetando o resultado de uma representação em EDM satisfatória que suporte a missão da plataforma. Por isso, a Europeana constantemente despende esforços para criar e disponibilizar uma documentação que auxilie os parceiros a compreender e atender aos requisitos necessários.

Contudo, para algumas instituições, seguir tais requisitos, nem sempre é algo simples de realizar, pois criar metadados interoperáveis e vinculáveis com qualidade e consistência é uma tarefa que exige investimento, especialização e ferramentas adequadas.

Deste modo, percebe-se que além da variedade de formatos, a variedade da qualidade dos dados é outro fator que afeta a representação dos dados de patrimônios culturais. Quando um conjunto de dados fornecidos apresenta deficiências que comprometem os aspectos semânticos da coleção ou não estão de acordo com as necessidades especificadas, os dados que estão ausentes ou insuficientes devem ser preenchidos de modo pertinente. Nesse caso, a veiculação automática de dados é uma solução. Essa solução, porém, traz outros desafios, como apoiar e incentivar às instituições provedoras para que contribuam com a possibilidade de trocar dados ao mesmo tempo em que aceitem reutilizar e vincular dados que não são próprios. 
Apesar disso, a Europeana tem conseguido disponibilizar um vasto conteúdo sobre patrimonios culturais em meio digital a partir de uma abordagem que contempla a riqueza e a variedade caracteristica dos dados de patrimonios culturais. Portanto, a representação em EDM vai além da atribuição de metadados padronizados em estruturas monolíticas. O modelo, ao contrário, garante a integração com diversos outros elementos descritivos que promovem uma rica contextualização semântica, fornecendo classes auxiliares para especificar metadados.

\section{Considerações Finais}

No cenário em que o volume de dados cresce em uma escala muito maior que o seu tratamento, o Europeana Data Model (EDM) configura-se com uma ferramenta de grande importância para o domínio dos patrimônios culturais, dada a sua funcionalidade que possibilita uma representação abrangente por meio da integração com diversos provedores de dados, promovendo uma rica contextualização semântica.

Com o EDM, a Europeana se torna uma plataforma de referência no domínio dos patrimônios culturais, não só por ser uma fonte que reúne e dá acesso um vasto conteúdo informacional, mas principalmente, porque configurase como um espaço de informações que interage com a Web e com os princípios do Linked Open Data, formando um rede de dados de alta qualidade que integra diversos recursos informacionais e elementos descritivos. Além disso, seu potencial está na possibilidade de disseminar a terceiros esse conteúdo de modo estruturado, promovendo amplo reuso e acesso aos dados.

Ao representar os dados do domínio de patrimônios culturais no EDM, a Europeana consegue contemplar a ampla variedade de tipos de dados que caracteriza esse domínio, considerando os possíveis relacionamentos culturalmente relevantes dessas coleções e ainda atendendo às diversas solicitações das comunidades de usuários.

Nesse sentido, destaca-se a modelagem de dados, pois é nesse processo fundamentado em princípios e requisitos, que o modelo pauta seu funcionamento e se torna flexível, descentralizado e adaptável a diversas 
aplicações. Tais características são o que tornam o modelo um instrumento de grande potencial para a organização e representação da informação em ambientes digitais, possibilitando contemplar as necessidades informacionais das comunidades de usuários.

Portanto, um modelo de dados, tal como o EDM, pode viabilizar que as necessidades de representação de coleções heterogêneas de patrimônios culturais sejam atendidas devido a sua capacidade de apreender o contexto semântico a qual um conjunto de dados pertence ou está relacionado, dando condições de garantir a persistência dos conceitos e das relações dos recursos de patrimônios culturais nos ambientes informacionais.

\section{Financiamento}

Coordenação de Aperfeiçoamento de Pessoal de Nível Superior (CAPES).

\section{Referências}

ALVES, R. C. V. Web semântica: uma análise focada no uso de metadados. 2005. Dissertação (Mestrado em Ciência da Informação) - Faculdade de Filosofia e Ciências, Universidade Estadual Paulista, Marília, 2005. 132 f. Disponível em: https://www.marilia.unesp.br/Home/PosGraduacao/CienciadaInformacao/Dissertacoes/alves_rachel.pdf. Acesso em: 30 jan. 2020.

\section{ALVES, R. C. V. Metadados como elementos do processo de catalogação.} 2010. 134 f. Tese (Doutorado em Ciência da Informação) - Faculdade de Filosofia e Ciências, Universidade Estadual Paulista, Marília, 2010. 132 f. Disponível em: https://www.marilia.unesp.br/Home/PosGraduacao/CienciadaInformacao/Dissertacoes/alves_rachel.pdf. Acesso em: 30 jan. 2020.

ARAKAKI, F. A. Linked data: ligação de dados bibliográficos. 2016. Dissertação (Mestrado em Ciência da Informação) - Universidade Estadual Paulista 'Júlio de Mesquita Filho', Marília/SP, 2016. 144 f. Disponível em: https://repositorio.unesp.br/handle/11449/147979. Acesso em: 30 jan. 2020.

BERNERS-LEE, T.; HENDLER, J.; LASSILA, O. The semantic web. Scientific American, New York, May, 2001, p. 29-37. Disponível em: https://www.ida.liu.se/ evabl45/files/bernersLee01Semantic.pdf. Acesso em: 30 jan. 2020.

CASTRO, F. F.; SIMIONATO, A. C.; ZAFALON, Z. R. Aspectos relacionais entre ontologia e metadados: considerações interdisciplinares. In: ENCONTRO 


\section{NACIONAL DE PESQUISA EM CIÊNCIA DA INFORMAÇÃO, 17,}

Salvador, 2016. Anais [...]. Salvador: Universidade Federal da Bahia, 2016.

Disponível em: http://www.academia.edu/download/51348047/2016_-

_Enancib_-_Aspectos_relacionais_entre_ontologias_e_metadados.pdf. Acesso em: 30 jan. 2020.

CHARLES, V.; ISAAC. A. Enhancing the Europeana Data Model (EDM). [S.l.]: Europeana Foundation, 2015. 21 f. Disponível em: https://pro.Europeana.eu/files/Europeana_Professional/Publications/EDM_Whit ePaper_17062015.pdf. Acesso em: 30 jan. 2020.

CONCORDIA, C.; GRADMANN, s.; SIEBINGA, S. Not (just) a repository, nor (just) a digital library, nor (just) a portal: a portrait of europeana as an API. In: WORLD LIBRARY AND INFORMATION CONGRESS IFLA GENERAL CONFERENCE AND COUNCIL, 75, 2009. Milão, Itália. Proceedings [...] Milão, Itália: IFLA, 2009. 13 f. Disponível em: https://www.ifla.org/pastwlic/2009/193-concordia-en.pdf. Acesso em: 30 jan. 2020.

CONEGLIAN, C. S.; SANTAREM SEGUNDO, J. E. Europeana no linked open data: conceitos de web semântica na dimensão aplicada das humanidades digitais. Encontros Bibli: Revista Eletrônica de Biblioteconomia e Ciência da Informação, Florianópolis, v. 22, n. 48, 2017. Disponível em: http://www.brapci.inf.br/v/a/22274. Acesso em: 30 jan. 2020.

CRUZ, I. F.; XIÃO, H. The role of ontologies in data integration. Engineering intelligent systems for electrical engineering and communications, Bentley, WA, v. 13, n. 4, p. 245, 2005. Disponível em: https://www.cs.uic.edu/ advis/publications/dataint/eis05j.pdf. Acesso em: 30 jan. 2020.

CUNHA, D. R. B; SOUZA, D.; LÓSCIO, B. F. Linked Data: da web de documentos para a web de dados. In: SANTANA, A. M., et al. Livro texto dos minicursos ERCEMAPI, SBC: Teresina, BR, p. 79-99, 2011.

COYLE, K. FRBR, before and after: a look at our bibliographic models. Chicago: American Library Association, 2016.

DOERR, M. The CIDOC conceptual reference module: an ontological approach to semantic interoperability of metadata. AI Magazine, Palo Alto, v. 24, n. 3, p. 75-75, 2003. Disponível em: https://www.aaai.org/ojs/index.php/aimagazine/article/view/1720. Acesso em: 14 jan. 2020.

DOERR, M. et al. The Europeana Data Model (EDM). IFLA General Conference and Assembly, 76, 2010. 12 f. Anais [...] Gothenburg: IFLA, 2010. Disponível em:

https://www.researchgate.net/publication/303058300_The_Europeana_Data_Mo del_EDM. Acesso em: 14 jan. 2020. 
DUBLIN CORE METADATA GLOSSARY. Using Dublin Core. [S.l.]:

DCMI, 2020. Disponível em: https://www.dublincore.org/specifications/dublincore/usageguide/2001-04-12/glossary/. Acesso em: 20 abr. 2020.

DUVAL, E. et.al. Metadata principles and practicalities. D-Lib, United States, v. 8, n. 4, 2002. Disponível em:

http://www.dlib.org/dlib/april02/weibel/04weibel.html. Acesso em: 20 abr. 2020.

EUROPEANA. Europeana Data Model primer. Dublin: Europeana Professional Website, 2013. 35 f. Disponível em:

https://pro.Europeana.eu/files/Europeana_Professional/Share_your_data/Techni cal_requirements/EDM_Documentation/EDM_Primer_130714.pdf. Acesso em: 14 jan. 2020.

EUROPEANA. Definition of the Europeana Data Model v5.2.8. [S.l.]: EDM, 2017. Disponível em:

https://pro.Europeana.eu/files/Europeana_Professional/Share_your_data/Techni cal_requirements/EDM_Documentation//EDM_Definition_v5.2.8_102017.pdf. Acesso em: 14 jan. 2020.

EUROPEANA. Europeana Publishing Guide. [S.l.]: EDM, 2019. Disponível em: https://pro.europeana.eu/post/publication-policy. Acesso em: 29 abr. 2020.

FUSCO, E. Modelos conceituais de dados como parte do processo da catalogação: perspectiva de uso dos FRBR no desenvolvimento de catálogos bibliográficos digitais. 2010. 249 f. Tese (Doutorado em Ciência da Informação) - Faculdade de Filosofia e Ciências, Universidade Estadual Paulista, Marília, 2010. 248 f. Disponível em: https://www.marilia.unesp.br/Home/PosGraduacao/CienciadaInformacao/Dissertacoes/fusco_e_do_mar.pdf. Acesso em: 14 jan. 2020.

HYVÖNEN, E. Publishing and using cultural heritage linked data on the semantic web. EUA: Morgan \& Claypool Publishers, 2012.

LISBOA FILHO, J.; IOCHPE, C. Um estudo sobre modelos conceituais de dados para projeto de bancos de dados geográficos. Revista IP-Informática Pública, Belo Horizonte, v. 1, n. 2, p. 67-90, 1999. Disponível em: http://www.ip.pbh.gov.br/ANO1_N2_PDF/ip0102lisboafilho.pdf. Acesso em: 20 jan. 2020.

LIU, D.; BIKAKIS, A.; VLACHIDIS, A. Evaluation of Semantic Web ontologies for modelling art collections. Springer International Publishing, Nicosia, Cyprus, 2017. Disponível em: https://doi.org/10.1007/978-3-31967162-8_34. Acesso em: 14 jan. 2020. 
MARCONDES, C. H. Interoperabilidade entre acervos digitais de arquivos, bibliotecas e museus: potencialidades das tecnologias de dados abertos interligados. Perspectivas em Ciência da Informação, Belo Horizonte, v. 21, n. 2, p. 61-83, jun. 2016. Disponível em: http://portaldeperiodicos.eci.ufmg.br/index.php/pci/article/view/2735. Acesso em: 30 jan. 2020.

MARCONDES, C. H. Relacionamentos culturalmente relevantes para interligar objetos do patrimônio digital na Web usando tecnologias de dados interligados. In: ENCONTRO NACIONAL DE PESQUISA EM CIÊNCIA DA INFORMAÇÃO, 19, 2018, Londrina, PR. Anais [...]. Londrina, PR: Universidade Estatual de Londrina, 2018. Disponível em:http://hdl.handle.net/20.500.11959/brapci/102416. Acesso em: 30 jan. 2020.

PETRAS, V. et al. Europeana: a search engine for digitised cultural heritage material. Datenbank-Spektrum, Leipzig, v. 17, n. 1, p. 41-46, 2017. Disponível em: https://doi.org/10.1007/s13222-016-0238-1. Acesso em: 20 abr. 2020.

POMERANTZ, J. Metadata. Cambridge, Mass: MIT Press, 2015.

PURDAY, J. Think culture: Europeana.eu from concept to construction. Bibliothek Forschung und Praxis, Berlin, v. 33, n. 2, p. 170-180, 2009. Disponível em: https://doi.org/10.1515/bfup.2009.018. Acesso em: 30 jan. 2020.

SOUZA, R. R.; ALVARENGA, L. A Web Semântica e suas contribuições para a ciência da informação. Ciência da Informação, Brasília, v. 33, n. 1, p. 132141, 2004. Disponível em: http://www.scielo.br/pdf/ci/v33n1/v33n1a16. Acesso em: 14 jan. 2020.

RAMALHO, R. A. S. Web Semântica: aspectos interdisciplinares da gestão de recursos informacionais no âmbito da Ciência da Informação. 2006. Dissertação (Mestrado em Ciência da Informação). Faculdade de Filosofia e Ciências Universidade Estadual Paulista, Marília, 2006. 120 f. Disponível em: https://www.marilia.unesp.br/Home/PosGraduacao/CienciadaInformacao/Dissertacoes/ramalho_ras_me_mar.pdf. Acesso em: 30 jan. 2020.

RDF WORKING GROUP. RDF: resource description framework. [S.l.]: W3C, 2014. Disponível em: http://www.w3.org/RDF/. Acesso em: 30 jan. 2020.

RILEY, J. Understanding metadata: what is metadata, and what is it for? Baltimore: National Information Standards Organization (NISO), 2017. Disponível em: http://www.niso.org/apps/group_public/download.php/17446/Understanding\%2 0Metadata.pdf. Acesso em: 30 jan. 2020. 
SANTAREM SEGUNDO, Jose Eduardo. Web semântica, dados ligados e dados abertos. Tendências da Pesquisa Brasileira em Ciência da Informação, João Pessoa, v. 8, n. 2, 2015. Disponível em:

https://www.brapci.inf.br/index.php/article/download/43737. 30 jan. 2020.

SOUZA, R. R.; ALVARENGA, L. A Web semântica e suas contribuições para a ciência da informação. Ciência da Informação, Brasília, v. 33, n. 1, p. 132-141, jan./abril 2004. Disponível em:

http://www.scielo.br/pdf/ci/v33n1/v33n1a16. Acesso em: 30 jan. 2020.

WINER, D.; ROCHA, I. E. Europeana: um projeto de digitalização e democratização do patrimônio cultural europeu. Patrimônio e Memória, Assis, v. 9, n. 1, 2013. Disponível em:

http://pem.assis.unesp.br/index.php/pem/article/view/327. Acesso em: 14 jan. 2020.

ZENG, M. L.; QIN, J. Metadata. 2. ed. Chicago, IL: ALA Neal-Schuman, 2016.

\title{
Representation of cultural heritage in digital platforms: the Europeana data model
}

\begin{abstract}
In order to represent relationships and entities in an abstract way, data models are highlighted as best practices in the processes of analysis and representation of information, specifically in the planning and development of interoperable and persistent systems. In this sense, a study is presented on the Europeana Data Model (EDM), a data model developed by the Europeana platform based on semantic technologies and the principles of Linked Open Data. Through a qualitative, exploratory, bibliographic and documentary research, it is discussed how a data model, such as EDM, allows the needs of informational representation of cultural heritage data collections to be met in the Web environment. The aim of the study was to analyze the structure and representation proposed by Europeana for collections of cultural heritage. As a result, data modeling stands out, which is the process by which EDM guide its operation, enabling information needs to be contemplated. Finally, we conclude that the importance of data models, such as EDM, lies in the possibility of understanding the semantic context to which a set of cultural heritage data belongs, guaranteeing the persistence of its concepts and relationships in the environments informational.
\end{abstract}

Keywords: Data Model. Metadata. Cultural heritage. Europeana. EDM.

Recebido: 04/02/2020

Aceito: $12 / 05 / 2020$ 


\section{Declaração de autoria}

Concepção e elaboração do estudo: Maria Lígia Triques e Ana Carolina Simionato Arakaki

Coleta de dados: Maria Lígia Triques e Ana Carolina Simionato Arakaki

Análise e discussão de dados: Maria Lígia Triques e Ana Carolina Simionato Arakaki

Redação e revisão do manuscrito: Maria Lígia Triques e Ana Carolina Simionato Arakaki

\section{Como citar}

TRIQUES, Maria Lígia; ARAKAKI, Ana Carolina Simionato. Representação de patrimônios culturais em plataformas digitais: o modelo de dados da Europeana. Em Questão, Porto Alegre, v.27, n. 1, p. 185-209, 2021. Doi: http://dx.doi.org/10.19132/1808-5245271.185-209

${ }^{1}$ Uma API, consistem em um conjunto de funções, que podem ser usadas para interagir com um programa de computador, possibilitando que um usuário exporte dados ou importe dados de um serviço da Web (POMERANTZ, 2015).

2 Conjunto de elementos de metadados, políticas e diretrizes definidas para um aplicativo específico. Os elementos podem ser de um ou mais conjuntos de elementos, permitindo que um determinado aplicativo atenda a seus requisitos funcionais usando metadados de vários conjuntos de elementos, incluindo conjuntos definidos localmente (DUBLIN CORE METADATA GLOSSARY, 2020, não paginado, tradução nossa).

3 Estruturas caracterizadas pela capacidade dos metadados serem combinados de maneira sintática e semanticamente interoperável de modo que novas estruturas possam ser criadas com base em padrões já estabelecidos (DUVAL et al. 2002). 